\title{
Design and beam dynamics simulations of an $S$-band photocathode rf gun
}

\author{
Arvind Kumar, K. K. Pant, and S. Krishnagopal \\ Beam Physics \& FEL Laboratory, Centre for Advanced Technology, Indore 452013, India
}

(Received 13 May 2002; published 17 October 2002)

\begin{abstract}
We are building an $S$-band photocathode $\mathrm{rf}$ gun as an injector to a $30 \mathrm{MeV}$ electron linac for FEL applications. Here we discuss details of design simulations performed using SUPERFISH and GDFIDL and compare with results of cold tests performed on prototype cells of the photocathode $\mathrm{rf}$ gun. We also discuss beam dynamics simulations performed using PARMELA and report results from simulations to achieve a normalized transverse rms emittance of about $1 \pi \mathrm{mm}$ mrad for a 10 ps pulse with $1 \mathrm{nC}$ charge in the presence of a solenoid magnetic field used for emittance compensation.
\end{abstract}

DOI: 10.1103/PhysRevSTAB.5.103501

PACS numbers: 41.75.Lx, 29.27.Fh

\section{INTRODUCTION}

There is growing interest in sources of high-brightness beams in recent years on account of their suitability for applications like x-ray free-electron lasers and linear colliders. Laser photocathode $\mathrm{rf}$ guns have proved to be promising candidates for the generation of such beams. A high accelerating field gradient $(\sim 150 \mathrm{MV} / \mathrm{m})$ in the laser photocathode rf gun accelerates electrons to relativistic speed within a very short distance, thereby reducing the Coulomb repulsion between the particles in the beam.

The first laser driven photocathode rf gun was proposed in the mid-1980s [1], following which many such structures have been studied, developed, and are currently operating in many laboratories around the world [2-4]. Our $S$-band photocathode gun is similar to the BNL/ SLAC/UCLA 1.6 cell structure [5] and is designed to resonate at $2856 \mathrm{MHz}$ in the $\pi$ mode. It will serve as an injector to a $30 \mathrm{MeV}$ electron linac being developed at our laboratory for free-electron laser applications. The photocathode rf gun is designed to give an electron beam of $1 \mathrm{nC}$ charge at around 4-5 MeV [6]. For further acceleration of the beam, an $S$-band plane wave transformer (PWT) linac structure [7] has been built and is currently being tested. A copper photocathode forms one wall of the half-cell of the laser photocathode rf gun structure. The drive laser system consists of a high stability Nd:VAN laser oscillator (GE-100 from TimeBandwidth), along with the CLX-1000 timing stabilizer, giving 7 ps pulses at a repetition rate of $102 \mathrm{MHz}$, with a timing jitter better than $3 \mathrm{ps}$, and an amplitude jitter better than $1 \%$ rms. The laser amplifier to be used for delivering $5 \mathrm{~mJ}$ in the $\mathrm{UV}$ is presently being developed. The gun will be powered by a $15 \mathrm{MW}$ microwave system, which will be synchronized with the laser system.

In the next Section, we discuss results of the 2D and 3D electromagnetic field solver codes SUPERFISH [8] and GDFIDL [9] used to generate a tolerance map for the photocathode rf gun structure. In Sec. III, we discuss results of cold tests performed on a prototype of the $\mathrm{rf}$ gun cells and compare these results with those obtained from the simulations. We also briefly discuss some fabrication issues for the structure. In Sec. IV, we discuss the beam dynamics simulations of the photocathode rf gun using PARMELA to study the emittance compensation and other beam properties of the beam. Section V describes the conclusions and our plans for the future.

\section{ELECTROMAGNETIC FIELD SIMULATIONS}

The preliminary design and numerical calculation of the $\mathrm{rf}$ parameters of the photocathode rf gun was performed using the 2D and 3D electromagnetic field solver codes SUPERFISH and GDFIDL, respectively. SUPERFISH assumes symmetry around the axis and hence cannot be used for determining the effects of asymmetric features in the photocathode rf gun, like port openings for $\mathrm{rf}$ power, vacuum pumps, and laser injection and extraction. Hence, for comparison of SUPERFISH results, and to evaluate the effect of port openings on the resonant frequency, SUPERFISH simulations were followed by 3D simulations using GDFIDL. Since the photocathode rf gun structure is cylindrically symmetric without the port openings, 2D simulations using SUPERFISH give a fairly true idea of the electrical properties of the structure. Table I shows the electrical parameters of the photocathode rf gun structure calculated using GDFIDL and SUPERFISH.

The simulation study performed for tolerance mapping shows that the photocathode gun is very sensitive to perturbations in the structure dimensions. Hence, fabrication of the photocathode rf gun is constrained by strict machining tolerances, typically of the order of 10-30 $\mu \mathrm{m}$. Simulations performed to study the effect of the port openings show a perturbation of a few $\mathrm{MHz}$ on the resonant frequency of the structure. There are limited mesh points in GDFIDL; hence, the results of 3D simulations include round off errors caused by considering the curved geometry of the gun as a mesh unit dimension. This may lead to a small deviation of the simulated results from the measured values. Our experience and a comparison of the simulated and measured results show that this deviation is typically less than $0.5 \%$. 
TABLE I. Electrical parameters of the photocathode rf gun calculated using SUPERFISH and GDFIDL.

\begin{tabular}{lccc}
\hline \hline \multicolumn{1}{c}{ rf parameters } & SUPERFISH & $\begin{array}{c}\text { GDFIDL } \\
\text { (Without ports) }\end{array}$ & $\begin{array}{c}\text { GDFIDL } \\
\text { (With ports) }\end{array}$ \\
\hline Resonant frequency $(\mathrm{MHz})$ & 2856 & 2856 & 2862 \\
Quality factor & 15348 & 13831 & 17300 \\
Effective shunt impedance $(\mathrm{M} \Omega / \mathrm{m})$ & 17 & 25 & 28 \\
Characteristic impedance $(\Omega)$ & 171 & 268 & 245 \\
\hline \hline
\end{tabular}

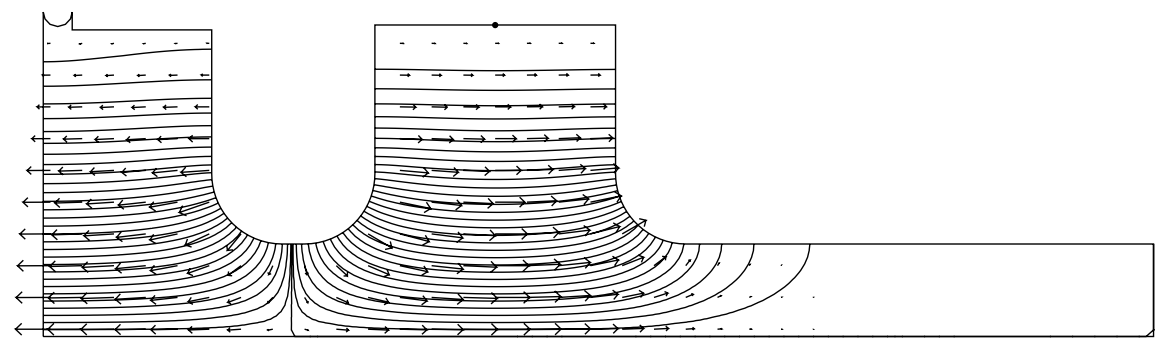

FIG. 1. Field contour plots of photocathode rf gun in the " $\pi$ " mode.

The electromagnetic field in the two cells of the photocathode rf gun is coupled through an aperture at the center of the disk separating the two cells. The size of the aperture is decisive in determining the coupling of electromagnetic power between the two cells. The bandwidth of the gun is of the order of $3.5 \mathrm{MHz}$. For the resonant frequency of $2856 \mathrm{MHz}$, the cell-to-cell coupling is $\sim 0.12 \%$.

SUPERFISH simulations are very helpful in calculating the effect of the aperture size on the resonant frequency of the photocathode rf gun. An increase in the aperture size decreases the capacitance of the gun cell and hence increases the resonant frequency of the gun. The effect of the aperture radius on the resonant frequency of the gun is about $0.2 \mathrm{MHz} / \mathrm{mm}$.

The full-cell of the gun is half a wavelength long, having an electric field directed opposite to that in the half-cell as shown in Fig. 1. Simulations predict a huge perturbation in the resonant frequency of the gun due to perturbation in the full-cell diameter $(\sim 46.1 \mathrm{MHz} / \mathrm{mm})$, while a change in the cell length gives a frequency perturbation of about $12.5 \mathrm{MHz} / \mathrm{mm}$ in full-cell and about $10 \mathrm{MHz} / \mathrm{mm}$ in the half-cell. For the fabrication tolerances prescribed, this sensitivity translates to a perturbation of about $100-200 \mathrm{kHz}$, which has to be compensated by some tuning mechanism.

\section{COLD TESTS OF PROTOTYPE RF GUN CELLS}

Prototypes of the half- and full-cells of the photocathode gun were fabricated to the accuracies prescribed using a tolerance map obtained using SUPERFISH and GDFIDL. The fabrication of the first prototype cells of the rf gun was done on a high speed CNC lathe out of
OFE copper. Tolerances of better than $20 \mu \mathrm{m}$ and a surface finish of $80 \mathrm{~nm}$ CLA was achieved by employing a single crystal, single diamond tool. These prototypes were subsequently inspected on a coordinate measurement machine (Carl Zeiss), which showed that the fabrication of the half-cell was not as per the design due to machining errors. The fabricated prototype is smaller than the designed prototype by $6 \mathrm{~mm}$, implications of which are discussed later. The ports for the laser injection and extraction from the half-cell are at an angle of $67.5^{\circ}$ from the axis of the gun. The port openings for $\mathrm{rf}$ and vacuum in the full-cell are placed symmetrically to avoid exciting the dipole mode.

A pair of brazing prototypes with all ports was also made to determine the optimization of brazing cycles required for brazing the gun assembly. The filler alloy used in these trials was $\mathrm{Cu}-\mathrm{Ag}$ eutectic (72-28) in wire and foil forms. With appropriate fixturing, it was observed that all the joints on the gun body, excluding the cathode plate, could be brazed in a single cycle. However, to tune the coupling coefficient " $\beta$ " between the waveguide and the gun structure, it might be necessary to braze the waveguide and the vacuum port in a separate cycle. All stainless steel ports were electroplated to a thickness of around $20 \mu \mathrm{m}$ before brazing. The brazed joints were leak tested using an Alcatel make helium leak detector and were found to have a leak rate smaller than $10^{-9}$ Torr $1 / \mathrm{s}$.

The cold test measurements of the gun cells were performed using a Hewlett Packard Vector Network Analyzer (VNA, model HP 8753E) at input power levels of $10 \mathrm{~mW}$. The vector network analyzer can be used to measure the frequency spectrum as well as to get information on the phase of the electromagnetic wave in the 


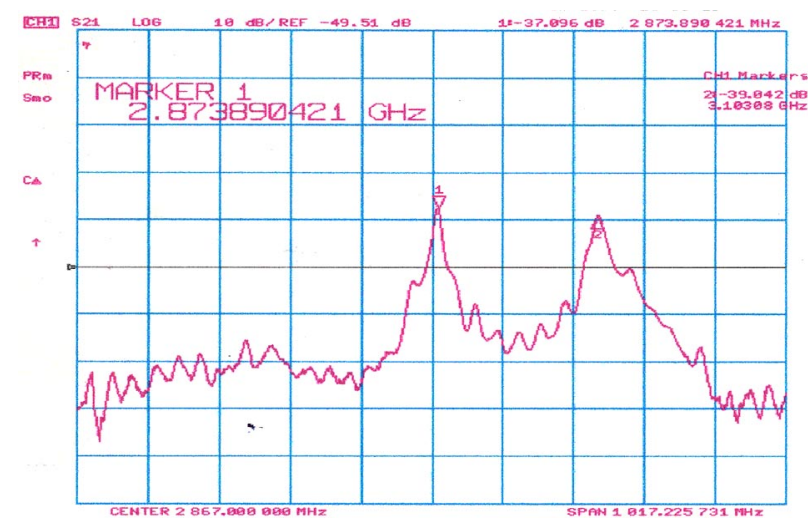

FIG. 2. (Color) Frequency spectrum of the coupled cells of the rf gun.

gun cells and in the transmission line for launching $\mathrm{rf}$ power into the gun. We performed frequency measurements on the prototypes of the gun cells in the transmission mode. In the cold test measurements, we used the rf and vacuum ports of the full-cell for injecting and sampling the rf power, respectively, using a loop antenna.

From mechanical engineering considerations, the halfand full-cells are both individually different from the ideal half- and full-cells studied in the simulations. Therefore, before cold testing the prototypes of the half- and full-cells, simulations were performed for the resonant frequency of the half- and full-cells as machined, which were then compared with results obtained from cold tests on the cells.

The GDFIDL simulations were performed using half symmetry of the structure with oblong port openings
$12.5 \mathrm{~mm}$ long and $9.5 \mathrm{~mm}$ wide. From symmetry, this is equivalent to considering this same dimension for all port openings. Figure 2 shows the frequency spectrum of the coupled cells obtained using the vector network analyzer. Table II shows a comparison of the measured resonant frequency of the gun cells to those predicted by the simulations.

As discussed earlier, the length of the half-cell was smaller than that of the simulated structure. Simulations of the actually machined structure show that the resonant frequency increases by about $30 \mathrm{MHz}$ giving a resonant frequency of $2883 \mathrm{MHz}$. The measured and simulation results are agreeing well within $0.6 \%$.

\section{BEAM DYNAMICS SIMULATIONS}

The beam dynamics of an intense, relativistic electron beam with a Gaussian distribution in space and time (truncated to flattop using PARMELA options) generated by a photocathode rf gun was studied using PARMELA. An emittance of about $1.1 \pi \mathrm{mm}$ mrad was obtained for the beam parameters given in Table III. PARMELA is a versatile multiparticle code that transforms the beam through a specified rf linac structure/transport line. It performs a 2D space-charge calculation and an optional 3D pointto-point space-charge calculation [10]. These simulations were performed with space-charge mesh of 20 radial grid lines and 400 longitudinal grid lines covering the electron beam fully. The postprocessor SF7 of SUPERFISH generates the TAPE7 field file for PARMELA by considering 10 radial increments and 60 longitudinal increments. We divided the photocathode rf gun length in 11 regions

TABLE II. Comparison of the measured and simulated results.

\begin{tabular}{lcccc}
\hline \hline & $\begin{array}{c}\text { SUPERFISH } \\
\text { Design values } \\
(\mathrm{MHz})\end{array}$ & $\begin{array}{c}\text { SUPERFISH } \\
(\text { Fabricated }) \\
(\mathrm{MHz})\end{array}$ & $\begin{array}{c}\text { GDFIDL } \\
\text { Fabricated }) \\
(\mathrm{MHz})\end{array}$ & $\begin{array}{c}\text { Cold tests } \\
(\mathrm{MHz})\end{array}$ \\
\hline Full-cell & 2789 & 2788 & 2789 & 2795 \\
Half-cell & 2854 & 2883 & 2881 & 2898 \\
Coupled cells & 2856 & 2883 & 2881 & 2875 \\
\hline \hline
\end{tabular}

TABLE III. PARMELA simulation parameters of the electron beam.

\begin{tabular}{lc}
\hline \hline PARMELA simulation parameters & Numerical values \\
\hline Distribution & Gaussian (both spatial and temporal) \\
Number of macroparticles & 10000 \\
Initial kinetic energy $(\mathrm{eV})$ & 0.3674 \\
Thermal emittance $(\pi \mathrm{mm} \mathrm{mrad})$ & 0 \\
Peak accelerating field $(\mathrm{MV} / \mathrm{m})$ & 147 \\
Beam injection rf phase $(\mathrm{degree})$ & 32 \\
Peak magnetic field $(\mathrm{kG})$ & 4.5 \\
Beam charge $(\mathrm{nC})$ & 1.0 \\
Longitudinal bunch length $(\mathrm{mm})$ & 3.0 \\
Radial bunch size $(\mathrm{mm})$ & 1.0 \\
\hline \hline
\end{tabular}


and generated a separate TAPE7 file for each region, to better model the electric field. These simulations were performed without considering thermal emittance and the Schottky effect, which is the reduction of the work function due to a large electric field gradient at cathode.

PARMELA simulations reveal that in the absence of a magnetic focusing field, a considerable amount of beam current gets lost in the gun for $\alpha<0.5$, where $\alpha=$ $e E_{0} / 2 m c^{2} k$ is a dimensionless parameter of the gun structure [11]. Here $e$ and $m$ are the charge and mass of the electron, respectively, $c$ is the velocity of light, $k$ is the wave number, and $E_{0}$ is the accelerating electric field gradient in the gun. The maximum possible field gradient in the gun is limited by dark currents and by electric breakdown in vacuum, given by the Kilpatrick criterion for a given frequency as $f(\mathrm{MHz})=1.64 E_{k}^{2} e^{-8.5 / E_{k}}$, where the peak field $E_{k}$ is in $\mathrm{MV} / \mathrm{m}$ [12].

Immediately after emerging from the cathode, electrons in the beam experience two forces: space-charge forces due to Coulomb repulsions and accelerating forces due to rf fields. For low accelerating field gradients, the space-charge forces dominate the beam dynamics causing a divergence of the beam. As the accelerating force increases, it can cause the electrons to get accelerated to high energies within short distances, thereby reducing the diverging effect of the space-charge forces. However, since the accelerating force is itself time dependent and since the gun is normally operated in the longitudinally stable region of the rf cycle, the emittance increase due to space-charge effect and reduction in space-charge effect due to $\mathrm{rf}$ forces neutralizes at some value of the accelerating field gradient in the gun, above which rf emittance also causes to deteriorate the beam emittance. In order to study this effect in the rf gun, we have performed PARMELA simulations by varying the peak solenoid field (Fig. 3) for different electric field gradients to achieve minimum transverse emittance as shown in Fig. 4. There are two dips in each curve in Fig. 4, which

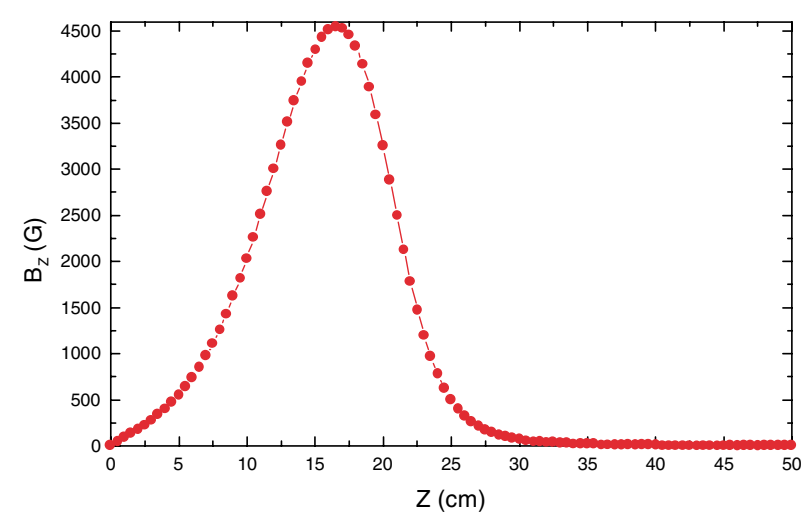

FIG. 3. (Color) Magnetic field distribution of the solenoid magnet.

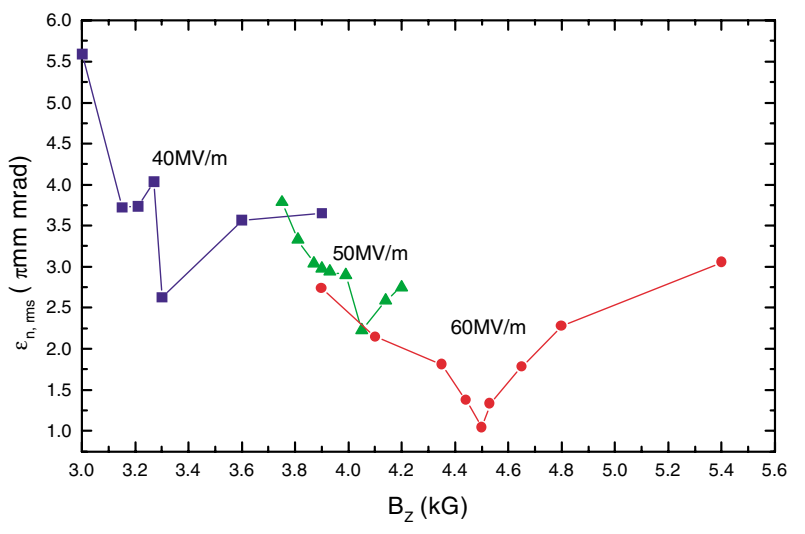

FIG. 4. (Color) Normalized transverse rms emittance variation with magnetic field for different electric field gradients.

shows the local minimum and the global minimum for each accelerating field. It is observed that at the end of the full-cell the electrons experience an extra transverse kick due to the rf field, causing an increase in the transverse emittance and this increases with increasing field gradient.

Figure 5 shows the variation of the transverse normalized rms emittance of the beam with the rf phase at the photocathode. It is observed that for a minimum transverse emittance, the laser beam should be launched at $\sim 32^{\circ}$ of the rf field, where $0^{\circ}$ corresponds to zero rf field gradient.

Figure 6 shows the bunch length variation with respect to the rf phase at the photocathode. The initial electron bunch length is chosen equal to the pulse length of the drive laser, typically $\sim 10 \mathrm{ps}$. The acceleration of electrons in the photocathode gun shrinks/expands the electron bunch length by an amount depending on the laser injection rf phase. The initial bunch length of $10 \mathrm{ps}$ shrinks down to $9.2 \mathrm{ps}$ at the exit of the full-cell for an injection phase of $25^{\circ}$, while for injection at $50^{\circ}$ of the $\mathrm{rf}$

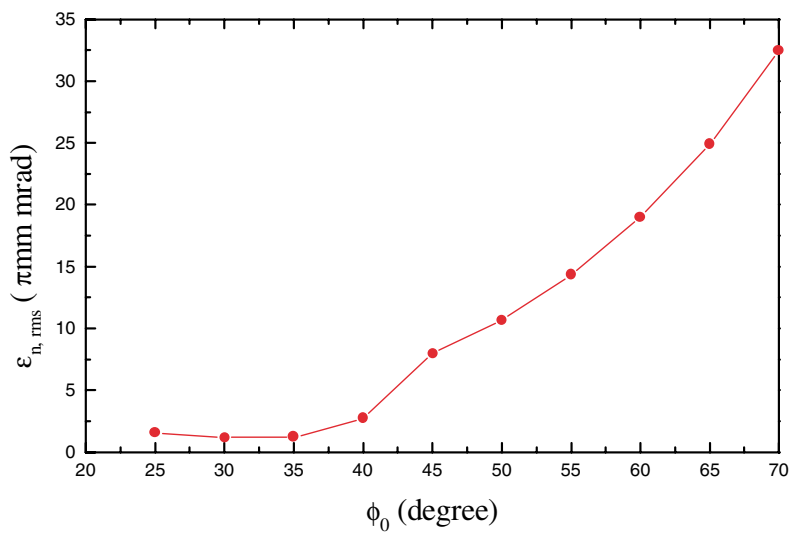

FIG. 5. (Color) Normalized transverse rms emittance vs rf phase at cathode. 


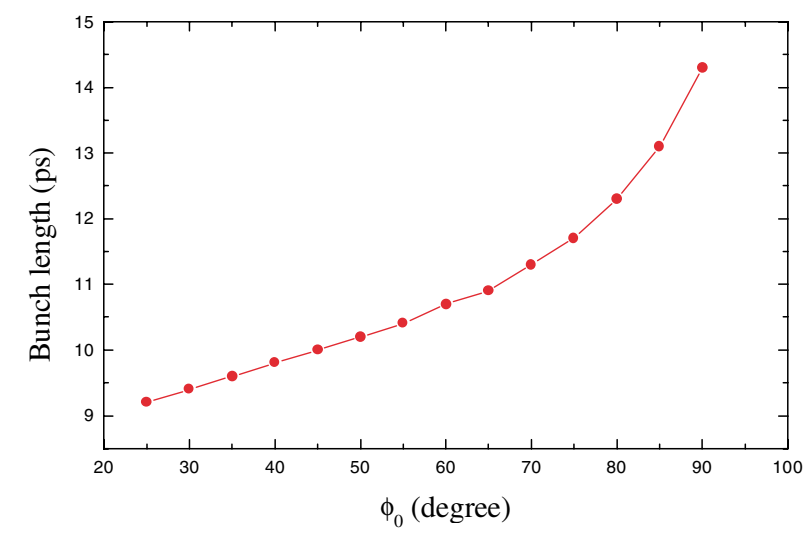

FIG. 6. (Color) Bunch length variation with $\mathrm{rf}$ phase at the cathode.

phase, the bunch length does not change much. The bunch is observed to expand up to $14.3 \mathrm{ps}$ at the $90^{\circ} \mathrm{rf}$ phase at photocathode.

The energy gain and spread with respect to the rf phase calculated numerically using PARMELA is shown in Fig. 7. These simulations show that a minimum energy spread $(0.9 \%)$ can be obtained by injecting the beam at an $\mathrm{rf}$ phase corresponding to a maximum energy gain in the gun, which occurs for an injection phase of $32^{\circ}$. For injection phases from $25^{\circ}$ to $90^{\circ}$, the energy spread varies from a minimum of $0.9 \%$ to a maximum of $35.0 \%$ of the synchronous particle energy gain.

The energy gain in the photocathode gun is directly proportional to the electric field gradient of the gun, as shown in Fig. 8. The peak accelerating field of operation of the photocathode $\mathrm{rf}$ gun normally is in the range of 60-150 MV/m.

The theory of the emittance compensation proposed by Serafini and Rosenzweig reveals that the beam dimensions should match the invariant beam envelope for effective emittance compensation [13]. In this process, a solenoid magnetic field is applied to mitigate space-

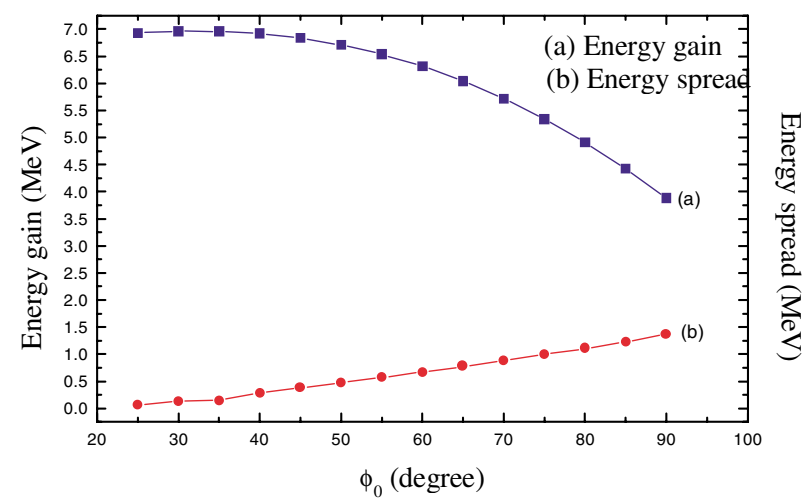

FIG. 7. (Color) Energy gain and energy spread in the photocathode gun as a function of the beam injection phase.

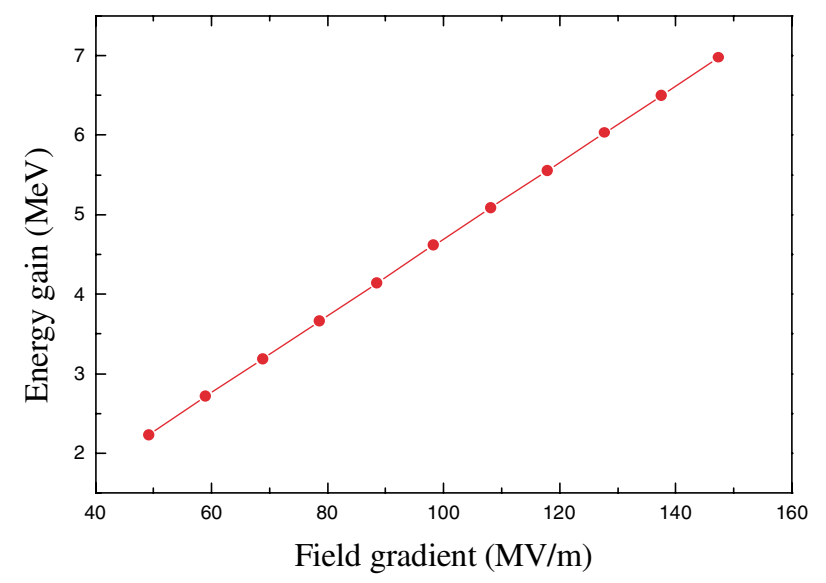

FIG. 8. (Color) Energy gain vs peak accelerating field gradient simulated using PARMELA.

charge effects of the beam emittance in the process of transport. Here, we report results of detailed simulations using PARMELA to study emittance compensation by scaling various parameters.

To suppress the effect of space-charge forces in transport and transverse rf kick at the exit of the gun, a solenoidal magnetic field is applied. In this way, the electron's motion becomes helical and transverse expansion of the beam comes to an end in the transport line, after which, the beam passes through the waist due to solenoid focusing. The focusing force neutralizes the space-charge force of the beam, and consequently the emittance and size of the beam are reduced to a minimum at the focal point of the solenoid.

By application of a solenoidal magnetic field, different transverse regions of the beam bunch experience different forces. Consequently, at a location in the transport line, the phase space projection of all these regions converges to the same angle resulting in a minimum beam

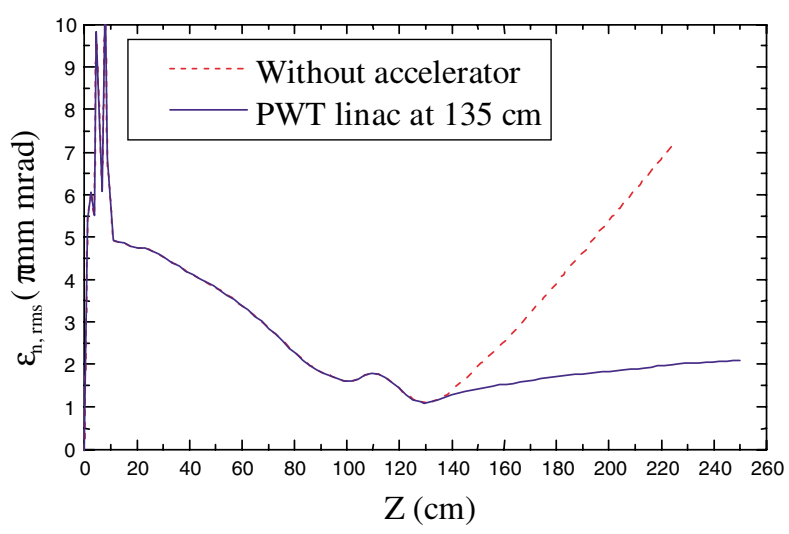

FIG. 9. (Color) PARMELA simulations for transverse emittance variation with distance, with a solenoid magnet field of $4.5 \mathrm{kG}$ at $16 \mathrm{~cm}$ from cathode and a 4-cell PWT linac at $135 \mathrm{~cm}$. 
emittance at this location [14]. At the location of the minimum emittance, we inserted a PWT linac structure to provide further acceleration to the beam, which further reduces the space-charge effect thereby helping in getting a lower emittance of the beam. In our simulations, we have applied a peak solenoid field of $4.5 \mathrm{kG}$ at the exit of the $\mathrm{rf}$ gun, $16 \mathrm{~cm}$ away from the cathode for an average field gradient of $60 \mathrm{MV} / \mathrm{m}$ in the $\mathrm{rf}$ gun. The solenoid magnetic field focuses the beam at $135 \mathrm{~cm}$ down the beam line and a PWT structure is inserted at this place for further acceleration of the beam. In this process, a minimum emittance of $\sim 1.1 \pi \mathrm{mm}$ mrad is achieved as shown in Fig. 9.

\section{CONCLUSION}

The rf photocathode gun has been designed using SUPERFISH and GDFIDL codes. We have fabricated prototype half-cell and full-cells, which were designed based on a detailed tolerance analysis of the rf gun structure. We get an agreement of better than $0.5 \%$ between simulations and the results of cold tests performed on these prototypes. Beam dynamics simulations predict a normalized rms transverse emittance of about $1.1 \pi \mathrm{mm} \mathrm{mrad}$ for the beam by using the emittance compensation technique using a solenoid at the exit of the gun. Beam dynamics simulations indicate that for an injection phase of $32^{\circ}$, an energy gain of about $7 \mathrm{MeV}$ is possible with a peak accelerating field gradient of the order of $147 \mathrm{MV} / \mathrm{m}$.

Based on the experience with the prototypes, the actual gun structure is currently under fabrication. We have commissioned a $10 \mathrm{MW}, S$-band, klystron based microwave system, which will be used to power the gun. A plane wave transformer linac system has also been fabricated and will soon be commissioned using the high power microwave line.

\section{ACKNOWLEDGMENTS}

We acknowledge the help rendered by Mr. S. Couksey and Mr. S. Jafar Ali in finalizing the AUTOCAD drawings and fabrication of the structure.

[1] J. S. Fraser, R. L. Sheffield, E. R. Gray, and G.W. Rodenz, IEEE Trans. Nucl. Sci. 32, 1791-1793 (1985).

[2] S. Anderson, J. Rosenzweig, K. Bishofberger, X. Ding, T. Holden, A. Murokh, C. Pellegrini, H. Suk, A. Tremaine, C. Clayton, C. Joshi, K. Marsh, and P. Muggli, in Proceedings of the 1999 Particle Accelerator Conference, New York (IEEE, Piscataway, NJ, 1999), p. 2006.

[3] Luca Serafini, Nucl. Instrum. Methods Phys. Res., Sect. A 341, 341-345 (1994).

[4] Kirk T. McDonald, IEEE Trans. Electron Devices 35, 2052 (1988).

[5] D. T. Palmer, Ph.D. thesis, Stanford University, 1998.

[6] Arvind Kumar, K. K. Pant, and S. Krishnagopal, CAT Report No. CAT/2001-04, 2001.

[7] Arvind Kumar, K. K. Pant, and S. Krishnagopal, Phys. Rev. ST Accel. Beams 5, 033501 (2002).

[8] K. Halbach and R. F. Holsiger, Part. Accel. 7, 213-222 (1976).

[9] W. Bruns, in Proceedings of the Particle Accelerator Conference, Vacouver, Canada, 1997 (IEEE, Piscataway, NJ, 1998), p. 2651.

[10] Computer code PARMELA, in Lloyd M. Young, Los Alamos National Laboratory Report No. LA-UR-961835, 1996.

[11] Kwang-Je Kim, Nucl. Instrum. Methods Phys. Res., Sect. A 275, 201-218 (1989).

[12] W. D. Kilpatrick, Rev. Sci. Instrum. 28, 824 (1957); T. J. Boyd, Jr., Los Alamos Group Report No. AT-1:82-28, 1982.

[13] Luca Serafini and James B. Rosenzweig, Phys. Rev. E 55, 7565 (1997).

[14] B. E. Carlsten, Nucl. Instrum. Methods Phys. Res., Sect. A 285 313-319 (1989). 